\title{
Unfolding the Pathogenesis of Systemic Sclerosis through Epigenomics
}

\author{
Xiuzhi $\mathrm{JIA}^{1}$, Hao $\mathrm{CHENG}^{2 *}$, Ying $\mathrm{XIAO}^{1 *}$ \\ ${ }^{1}$ Central Lab of Biomedical Research Center, Sir Run Run Shaw Hospital, School of Medicine, Zhejiang University. Hangzhou, Zhejiang Province, China. \\ 'Department of Dermatology, Sir Run Run Shaw Hospital, School of Medicine, Zhejiang University, Hangzhou, Zhejiang Province, China.
}

*Correspondence: chenghao1@zju.edu.cn; xiaoying.srr@zju.edu.cn https://doi.org/10.37175/stemedicine.v1i1.5

\begin{abstract}
As a group of autoimmune diseases, systemic sclerosis (scleroderma, SSc) is prominent in the imbalance of immune homeostasis, micro-vessels dominant obliteration, and the skin and/or internal organs fibrosis. Although the precise mechanisms are still unknown, increasing data have shown that epigenetic dysregulation, which can link genetics and environmental stress, represents a promising field in SSc investigation. The objective of this review is to sum up the current information on the epigenetic alteration in SSc, including DNA methylation, histone modification, and microRNA.
\end{abstract}

Keywords: Epigenetics · Methylation · Histone modification · microRNA · Systemic sclerosis

\section{Introduction}

Systemic sclerosis (scleroderma, SSc) was first reported by Carlo Curzio in 1753 (1), with the disease becoming well-documented by 1842 (2). As a rare connective tissue disease (ranging from 7 to 700 cases per million), SSc is characterized by vascular anomaly, chronic inflammation, and fibrosis process. Significant female bias (> 80\%) (3), racial discrepancy (4), and geographic clustering (5) are observed in SSc, which suggests that environmental, genetic, and hormonal factors may contribute to the initiation and development of this disease.

The precise mechanism of how these external environmental factors, such as silica, ketones, ultraviolet light, trichloroethylene, aromatic and chlorinated solvents, white spirits, and welding fumes, can induce an autoimmune attack is still unknown. The complexity of the issue is further enhanced by the possibility that environmental factors may not only induce cellular and tissue damages associated with both innate and adaptive immunity, but also alter fibroblasts and microvascular endothelial cell phenotype or function.

Without causing alterations in the DNA sequence, epigenetics can cause heritable phenotypic changes $(6,7)$, which is vital in the regulation of gene expression and development. Major mechanisms of epigenetic gene regulation, such as chromatin remodeling, histone modification, DNA methylation, transcriptional regulation

(C) The Author(s). 2019 This is an Open Access article distributed under the terms of the Creative Commons License (http://creativecommons.org/licenses/by/4.0/) which permits unrestricted use, distribution, and reproduction in any medium or format, provided the original work is properly cited. by non-coding RNA, and gene imprinting, can provide plausible links between environmental factors and disease predisposition and perpetuation. It is possible that epigenetics plays a vital role in the pathogenesis of SSc. It is worth noting that with the development of high throughput omics, it is testified that epigenetic mechanisms can lead to downstream effects on modulation of chromatin architecture and regulate gene transcription. As only a few epigenetic studies have been performed in SSc, the recent progress in DNA methylation, histone modification, and microRNAs are reviewed in this paper (Figure 1).

\section{DNA methylation}

Most DNA methylation occurs at the 5-position of cytosine residues in a short canonical sequence 5'-CG-3' $(\mathrm{CpG})$ enriched in promoter regions, which arises from de novo DNA methyltransferases (DNMTs), such as DNMT3A and DNMT3B during development. This methylation pattern could be inherited and maintained by epigenetic maintenance mediator, DNMT1, in proliferating cells (8). Hence, DNA methylation can be considered as a stable epigenetic mark that usually represses gene expression (9). Increasing investigations have indicated that DNA methylation dysregulation is involved in pathogenesis of SSc in fibroblasts, microvascular endothelial cells, and lymphocytes.

\section{DNA methylation in fibroblasts}

At a global level, BeadChip array analysis has identified 2710 differentially regulated $\mathrm{CpG}$ sites in SSc 


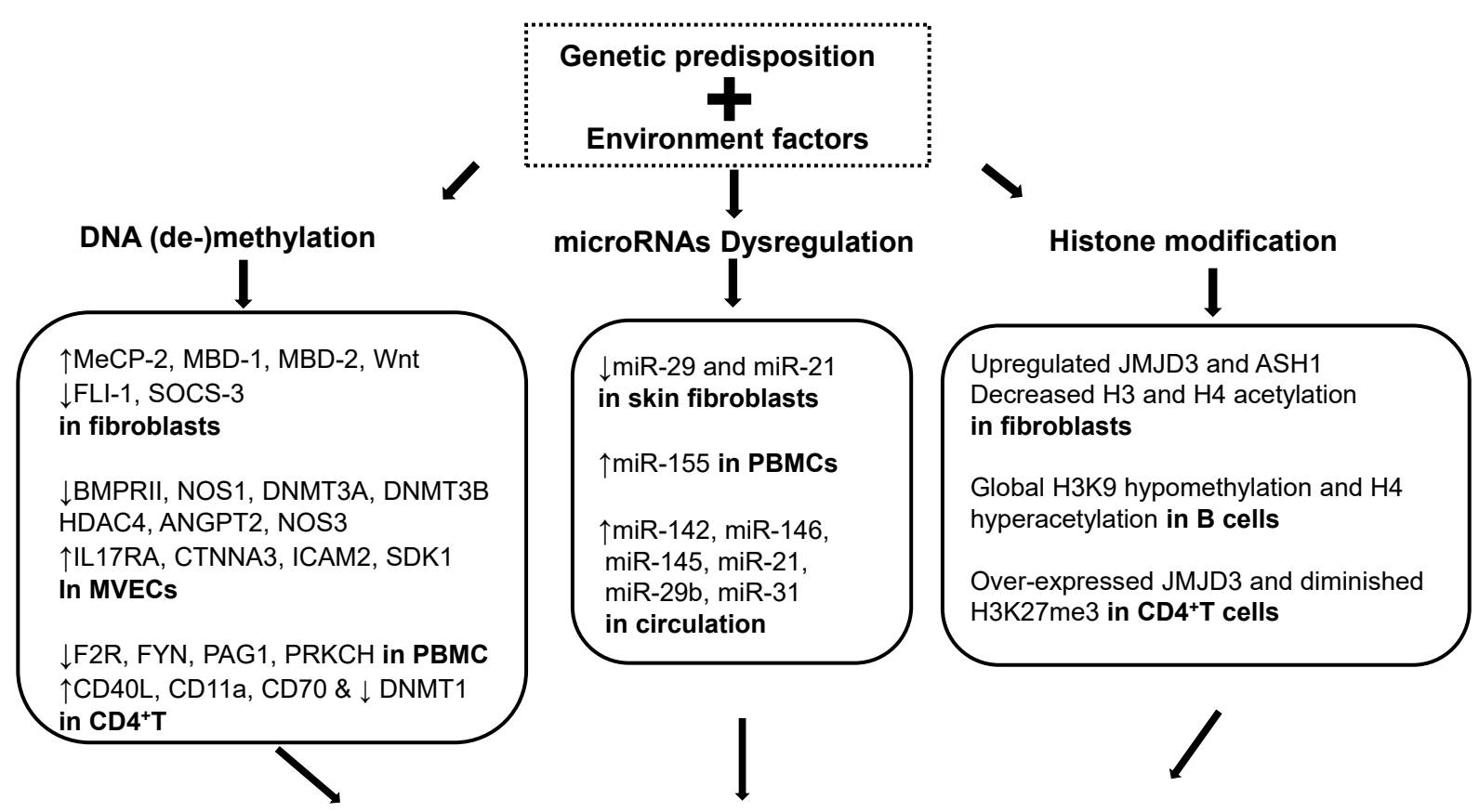

Fibroblast activation, vasculopathy, immune dysregulation

Figure 1. Possible roles of epigenetic mechanisms involved in systemic sclerosis pathogenesis. Genetic disposition and environmental factors can alter DNA (de-)methylation, histone modifications and microRNAs regulation, which can contribute to systemic sclerosis associated fibroblasts activation, vasculopathy, and immune dysregulation. Abbreviation: Methyl-CpG binding protein 2, MeCP-2; methyl-CpG DNA binding protein,MBD-1; Friend leukemia integration factor 1, FLI-1; bone morphogenic protein receptor II, BMPRII; nitric oxide synthase 1, NOS1; histone deacetylases 4, HDAC4; angiopoietin 2, ANGPT2; interleukin 17 receptor, IL-17RA; catenin alpha 3, CTNNA3; intercellular adhesion molecule 2, ICAM2; sidekick cell adhesion molecule 1, SDK1; coagulation factor II thrombin receptor, F2R; FYN proto-oncogene, Src family tyrosine kinase, FYN; phosphoprotein membrane anchor with glycosphingolipid microdomains 1 , PAG1; protein kinase C eta, PRKCH; DNA (cytosine-5)-methyltransferase 1, DNMT1; 3-Deazaneplanocin A, DZNep; JMJ domaincontaining protein 3, JMJD3.

fibroblasts when compared with healthy controls. It is further demonstrated that most of the affected $\mathrm{CpG}$ sites $(61 \%)$ are hypomethylated and associated with extracellular matrix metabolism (10). Methyl-CpG binding protein 2 (MeCP-2), methyl-CpG DNA binding protein (MBD)-1, and MBD-2 show increased expression, which can function as epigenetic maintenance mediators. All of these indicate that epigenetic inheritance is also involved in the development of SSc.

In addition to reduced acetylation of $\mathrm{H} 3$ and $\mathrm{H} 4$, several $\mathrm{CpG}$ islands within Friend leukemia integration factor 1 (FLI-1), a pro-fibrotic signaling cascade relevant transcription factor, are hypermethylated in both cultured primary SSc fibroblasts and skin biopsies compared with healthy control (11). Inhibition of DNMTs by 2-deoxy5-azacytidine (5-Aza) can remove methyl groups from $\mathrm{CpG}$ islands of FLI-1 promoter region and reactivate the expression of FLI-1 and down-stream type I collagen production in SSc fibroblasts. In addition to the aberrant activation of fibroblasts, epigenetic silencing of FLI-1 can cause SSc relevant vascular pathogenesis.

Suppressor of cytokine signaling 3 (SOCS-3), as an antifibrotic mediator and endogenous inhibitor of Janus kinase 2 (JAK2), can down-regulate transforming growth factor (TGF)- $\beta$ induced collagen secretion, fibroblast activation, myofibroblast differentiation, and fibrosis formation (12). SOCS-3 knockdown can activate JAK2/STAT3 signaling and induce the differentiation of fibroblasts into myofibroblasts. SOCS-3 is down-regulated in SSc fibroblasts through promotor hypermethylation, which may lead to persistent activation of fibroblasts and further fibrosis. The canonical Wnt signaling plays a central role in SSc fibrosis $(13,14)$. The balance lying between Wnt ligands and endogenous Wnt antagonists can regulate canonical Wnt signaling activation. In SSc, owing to overexpressed Wnt ligands and epigenetic silencing of Wnt antagonists, such as Dick-kopf1 (DKK1) and secreted frizzled-related protein-1 (SFRP1), such balance is shifted toward activation $(15,16)$. At the same time, 5-Aza treatment can increase DKK1 and SFRP1 expression, inhibit canonical Wnt signaling, and exert anti-fibrotic effects.

\section{DNA methylation in microvascular endothelial cells (MVECs)}

Bone morphogenic protein (BMP) can interact with bone morphogenic protein receptor II (BMPRII) to coordinate the survival, proliferation, and differentiation of SSc MVECs. A significantly decreased expression of BMPRII is observed in MVECs due to heavily methylated $\mathrm{CpG}$ sites in the promoter region of BMPRII after bisulfite conversion sequencing (17). The administration of 5-Aza to SSc MVECs can restore the decreased BMPRII expression and the impaired apoptotic response to oxidation injury and serum starvation. All of these indicate 
that the epigenetic modulation of BMPRII signaling might have a fundamental role in the sensitivity of MVECs to apoptosis and vasculopathy.

A genome-wide MVECs (isolated from seven diffuse cutaneous SSc patients) DNA methylation assay has identified 1,625 hypermethylated $\mathrm{CpG}$ sites (910 genes) and 830 hypomethylated $\mathrm{CpG}$ sites (485 genes), which show a significant negative correlation between gene expression and DNA methylation status. Among them, nitric oxide synthase 1 (NOS1), DNMT3A, DNMT3B, histone deacetylase 4 (HDAC4), and angiopoietin 2 (ANGPT2) are the common hypermethylated genes, while interleukin (IL)-17RA, catenin alpha 3 (CTNNA3), intercellular adhesion molecule 2 (ICAM2), and sidekick cell adhesion molecule 1 (SDK1) are the common hypomethylated genes. Of note, high methylation in the NOS3 promoter region can lead to reduced NOS3 expression in SSc MVECs, which can be reversed by the administration of 5-Aza (18).

\section{DNA methylation in peripheral blood mononuclear cells (PBMCs)}

Genome-wide DNA methylation and transcriptome integration analysis with a machine learning algorithm have identified six methylation-regulated differentially expressed genes in PBMCs of SSc, which can distinguish SSc from healthy control with 100\% accuracy. Among these six genes, Coagulation Factor II Thrombin Receptor (F2R), FYN Proto-Oncogene, Src Family Tyrosine Kinase (FYN), Phosphoprotein Membrane Anchor With Glycosphingolipid Microdomains 1 (PAG1), and Protein Kinase C Eta (PRKCH) are differentially expressed in SSc with interstitial lung disease compared to SSc without interstitial lung disease (19). The whole blood DNA methylation integrated analysis in discordant twins reveals that diffused cutaneous SSc-associated CpGs are enriched at the encyclopedia of DNA elements, roadmap, and blueprint-derived regulatory regions, which indicates a potential role in the initiation of this disease. Notably, the dominant enriched regions in macrophages and monocytes can act on fibrosis, indicating that the dysregulated cellular function can be related to altered epigenetic mechanisms in diffuse cutaneous SSc (20).

In $\mathrm{SSc} \mathrm{CD}^{+} \mathrm{T}$ cells, global hypomethylation can be attributed to the significantly decreased DNMT1 expression, which may alter the reactivation of endoparasitic sequences and lead to autoimmunity. It is intriguing to find the methylation divergence between fibroblasts and MVECs (increased methylation) and $\mathrm{CD} 4^{+} \mathrm{T}$ cells (decreased methylation). Although further investigation is required to understand the detailed mechanisms, it is reported that extracellular signalregulated kinase (ERK) defect may regulate T cell DNA methylation in arthritis and lupus (21). Such a mechanism may also function in $\mathrm{SSc} \mathrm{CD}^{+} \mathrm{T}$ cells.

Demethylation can be observed in the promoter of CD40L on the inactive $\mathrm{X}$ chromosome derived from female SSc $\mathrm{CD}^{+} \mathrm{T}$ cells, which indicates impaired DNA methylation maintenance and reactivation of the normally silenced X chromosome (22). Of note, CD40L expression is up-regulated in SSc fibroblasts obtained from affected $\mathrm{CD}^{+} \mathrm{T}$ cells and skin, especially in female SSc patients $(23,24)$. CD40L is testified to take part in fibrosis, adhesion of endothelial cells, and B cell activation. Such gender-biased epigenetic alteration can explain the female tendency in SSc.

Co-stimulation is fundamental to develop an optimized immune response. Demethylation of CD11a and CD70 promoter regions contributed to CD11a and CD70 overexpression in $\mathrm{CD}^{+} \mathrm{T}$ cells, which may also be involved in the development of SSc $(25,26)$. Furthermore, knockdown of SOCS-3 in T cells in the donor graft exacerbates sclerodermatous graft versus host disease $(27,28)$. All of these indicate that DNA methylation alteration can induce the development of SSc.

\section{Histone modifications}

As the essential component of the nucleosome, histones can be classified into four types (H2A, H2B, H3, and $\mathrm{H} 4$ ), which are known to be modified by acetylation and methylation to regulate chromatin architecture. Histone acetylation usually leads to transcriptional activation, while the inhibition or activation effect of histone methylation mainly depends on the position of lysine methylation. For example, H3K27 trimethylation (H3K27me3) could decrease the relevant gene expression, while histone $\mathrm{H} 3$ lysine 4 (H3K4) methylation can increase the associated gene expression (29). It is interesting to find that DNA methylation can lead to methyl binding domain (MBD) and further HDAC recruitment, which indicates the mechanical linkage of DNA methylation and histone modifications.

Treatment with trichostatin (TSA), an HDAC inhibitor available for the myelodysplastic disease, can attenuate the expression and accumulation of collagen I, extracellular matrix, and fibronectin in SSc fibroblast-induced skin fibrosis $(11,30,31)$. Similarly, TSA administration can increase $\mathrm{H} 3$ and $\mathrm{H} 4$ acetylation on the NOS3 promoter region accompanied by up-regulated NOS expression in SSc MVECs (32).

The pro-fibrotic transcription effects of 3-Deazaneplanocin A (DZNep, C-c3Ado), an inhibitor of histone methyltransferase enhancer of Zeste homolog 2 (EZH2), might result from its stimulation on Fos-related Antigen 2 (Fra2) to foster the release of tissue inhibitor of metalloproteinases (TIMP) and the promotion of fibroblast-to-myofibroblast differentiation (33). Fra2 transgenic mice would spontaneously develop skin and lung fibrosis, vascular smooth muscle proliferation, and pulmonary arteries obliteration. In line with these results, increased expression of Fra2 can be detected in skininfiltrating macrophages and perivascular regions of SSc patients (34).

Significantly decreased $\mathrm{H} 3$ and $\mathrm{H} 4$ acetylation and increased $\mathrm{H} 3 \mathrm{~K} 27 \mathrm{me} 3$ are detected on the Fli-1 promoter region of SSc fibroblasts, which could result in the inhibition of Fli-1. It is also demonstrated that H3K27me3 inhibition could stimulate collagen release in 
SSc fibroblasts $(27,35)$. Although H3K27me3 is considered stable, ubiquitously transcribed tetratricopeptide repeat on chromosome X (UTX) and JMJ domain-containing protein 3 (JMJD3) have recently been testified to have potent $\mathrm{H} 3 \mathrm{~K} 27$ demethylase activity $(36,37)$. A TGF- $\beta$ dependent JMJD3 upregulation and associated $\mathrm{H} 3 \mathrm{~K} 27 \mathrm{me} 3$ de-methylation might exert profibrotic functions in SSc. As a histone methyltransferase, absent, small or homeotic disc 1 (ASH1) can mediate H3K 4 methylation and regulate COL1A1 (profibrotic gene) and TGF- $\beta$ associated gene expression in hepatic fibrosis. Up-regulated ASH1 is observed in SSc fibroblasts, which indicates ASH1 may mediate the pathogenesis of SSc (29).

Global histone H3K9 hypomethylation and global histone $\mathrm{H} 4$ hyperacetylation are observed in SSc B cells compared with healthy control, which correlates with skin thickness and disease activity (38). Relatively low levels of $\mathrm{H} 3 \mathrm{~K} 27 \mathrm{me} 3$ can be observed in the $\mathrm{CD} 4^{+} \mathrm{T}$ cells of SSc patients when compared with healthy control. JMJD3 is over-expressed in $\mathrm{CD}^{+} \mathrm{T}$ cells of SSc patients, accompanied by lower levels of H3K27me3. All of these indicate that JMJD3 may be vital to decipher the mechanism related to histone modifications (39). Altered chromatin marks in SSc monocytes can be enriched with antiviral pathways, interferon, and immune system, and present with recurrent binding sites for interferon regulatory factor (IRF)-1 and signal transducer and activator of transcription (STAT)-1, which is correlated with their interferon signature.

\section{microRNA (miRNA, miR)}

As a small (about 22 nucleotides) non-coding RNA molecule, tissue-specific or cell intrinsic miRNA can function in RNA silencing and gene post-transcriptional regulation (40-42).

miR-29 is the first miRNA detected in the skin fibroblasts derived from SSc patients, whose downregulation contributes to the pathogenesis of SSc. Irritation of fibroblasts derived from normal skin with profibrotic molecules, such as platelet-derived growth factor (PDGF) and TGF- $\beta$, can decrease miR-29 secretion; while the restoration of miR-29 can reduce collagen release (43). TGF- $\beta$ can up-regulate miR-21 expression in SSc fibroblasts (44). The over-expression of miR-
21 can decrease SMAD Family Member 7 (Smad-7) expression, while miR-21 knockdown can increase Smad7 expression (45), which indicates that miR-21 can exert a pro-fibrogenic effect by negatively regulating Smad-7.

Nine pro-fibrotic miRNAs are upregulated, while 14 anti-fibrotic miRNAs are down-regulated within exosomes isolated from the serum of diffuse cutaneous SSc patients (Table 1). The dose-dependent paracrine pro-fibrotic effects of such miRNAs are also confirmed when cultured with normal human dermal fibroblasts to alter the pro-fibrotic gene expression and increase type I collagen production (46). It is worth noting that miR-92a level in serum is higher in SSc patients when compared with healthy control, and correlates with telangiectasia severity, but not with SSc activity (47). On the other hand, circulating miR-142, miR-146, miR-145, miR21 , miR-29b, and miR-31 do correlate with the severity of SSc (48-51). Moreover, miR-29a can directly downregulate mRNA and protein expression of type I and type III collagen. miR-155 expression in PBMC strongly correlates with lung function tests in SSc-associated interstitial lung disorder, and miR-155 knock-out mice develop milder lung fibrosis and survive longer (52). All of these indicate that some miRNAs, especially exosome derived, can be expected to serve as prognostic and diagnostic markers for precision medicine (53).

It is noteworthy that histone deacetylation and methylation also take part in the regulation of miRNA transcription (54). Such interactions should be regarded in the treatment of SSc with epigenetic modulators, whose effects are considered as the diffuse and unknown off-site effect. Exosomes can be utilized as cargos to transduce the inter-cellular information associated with epigenetic alteration, such as DNA methylation, histone modification, miRNA, and long intergenic non-coding RNA (LincRNA) (55-58), to reflect the status of donor cells and imprint recipient cells $(53,59)$. Although further refinements to exosome-based drug delivery systems are needed to meet the clinical scale, elaborate design can ensure direct and specific target, which will contribute to limit the diffuse effect of epigenetic modulators.

\section{Conclusions}

Epigenetics can imprint short-term signals or stress into longer-lasting, more stable, and inherited phenotypic

Table 1. Exosomes derived microRNA for SSc.

\begin{tabular}{|c|c|}
\hline Detected microRNAs & Function implication \\
\hline $\begin{array}{l}9 \text { upregulated exosomes-derived miRNAs (miR-21-5p, miR-503-5p, miR-155-5p, } \\
\text { miR-29a-3p, miR-17-5p, miR-let-7g-5p, miR-23b-5p, miR-150-5p, and miR-215-5p) }\end{array}$ & Profibrotic function \\
\hline $\begin{array}{l}14 \text { downregulated exosomes-derived miRNAs (miR-200a-3p, miR-140-5p, miR-92a- } \\
\text { 3p, miR-29b-3p, miR-223-3p, miR-26b-5p, miR-196a-5p, miR-145-5p, miR-200b-3p, } \\
\text { miR-let-7a-5p, miR-125b-5p, miR-133a-3p, miR-146a-5p, and miR-129-5p ) }\end{array}$ & Anti-fibrotic function \\
\hline
\end{tabular}
(scleroderma, SSc) patients, which has been reported by Wermuth PJ. et al (46). 
changes. Thus, epigenetic treatment strategies may be able to treat or even reverse tissue fibrosis through rescuing the imprinted genes. Exosome-based epigenetic modulator delivery maybe a new direction in SSc clinical practice.

\section{Acknowledgements}

Thank Chen Rui and Hao Zhang for their kindly help in the manuscript preparation process.

\section{Conflict of interest}

The authors declare that there are no conflicts of interest.

\section{Funding body}

This project is supported by the scientific research start-up funds for specially engaged employees of Sir Run Run Shaw Hospital (Ytp1902) and fund of the National Nature Science Foundation of China $(81660708,31900620)$.

\section{References}

1. Rose NR, Mackay IR. CHAPTER 1 - The immune response in autoimmunity and autoimmune disease. In: Rose NR, Mackay IR, editors. The Autoimmune Diseases II. Boston: Academic Press; 1992. p. 1-26.

2. Firestein GS, Kelley WN. Kelley's textbook of rheumatology. Philadelphia, PA: Elsevier/Saunders; 2013.

3. Gourier G, Therene C, Mazeas M, Abasq-Thomas C, Brenaut $E$, Huet $F$, et al. Clinical characteristics of pruritus in systemic sclerosis vary according to the autoimmune subtype. Acta Derm Venereol. 2018;98(8):735-41.

4. Mayes MD. Scleroderma epidemiology. Rheum Dis Clin North Am. 2003;29(2):239-54.

5. Valesini G, Litta A, Bonavita MS, Luan FL, Purpura M, Mariani M, Balsano F. Geographical clustering of scleroderma in a rural area in the province of Rome. Clin Exp Rheumatol. 1993;11(1):41-7.

6. Sen D, Keung AJ. Designing epigenome editors: considerations of biochemical and locus specificities. In: Jeltsch A, Rots MG, editors. Epigenome Editing: Methods and Protocols. New York, NY: Springer New York; 2018. p. 65-87.

7. Dupont C, Armant DR, Brenner CA. Epigenetics: definition, mechanisms and clinical perspective. Semin Reprod Med 2009;27(5):351-7

8. Richardson B. Primer: epigenetics of autoimmunity. Nat Clin Pract Rheumatol. 2007;3(9):521-7

9. Du J, Johnson LM, Jacobsen SE, Patel DJ. DNA methylation pathways and their crosstalk with histone methylation. Nat Rev Mol Cell Biol. 2015;16(9):519-32

10. Altorok N, Tsou P-S, Coit P, Khanna D, Sawalha AH. Genome-wide DNA methylation analysis in dermal fibroblasts from patients with diffuse and limited systemic sclerosis reveals common and subset-specific DNA methylation aberrancies. Ann Rheum Dis. 2015;74(8):1612-20.

11. Wang Y, Fan P-S, Kahaleh B. Association between enhanced type I collagen expression and epigenetic repression of the FLI1 gene in scleroderma fibroblasts. Arthritis Rheum. 2006;54(7):2271-9.

12. Dees C, Tomcik M, Palumbo-Zerr K, Distler A, Beyer C, Lang $\mathrm{V}$, et al. JAK-2 as a novel mediator of the profibrotic effects of transforming growth factor beta in systemic sclerosis. Arthritis Rheum. 2012;64(9):3006-15.

13. Bergmann C, Distler JHW. Canonical Wnt signaling in systemic sclerosis. Lab Invest. 2016;96(2):151-5.

14. Henderson WR, Jr., Chi EY, Ye X, Nguyen C, Tien Y-t, Zhou $B$, et al. Inhibition of Wnt/beta-catenin/CREB binding protein (CBP) signaling reverses pulmonary fibrosis. Proc Natl Acad Sci USA. 2010;107(32):14309-14.

15. Svegliati S, Marrone G, Pezone A, Spadoni T, Grieco A, Moroncini $\mathrm{G}$, et al. Oxidative DNA damage induces the
ATM-mediated transcriptional suppression of the Wnt inhibitor WIF-1 in systemic sclerosis and fibrosis. Sci Signal. 2014;7:ra84-ra.

16. Dees C, Schlottmann I, Funke R, Distler A, Palumbo-Zerr $\mathrm{K}$, Zerr $\mathrm{P}$, et al. The Wnt antagonists DKK1 and SFRP1 are downregulated by promoter hypermethylation in systemic sclerosis. Ann Rheum Dis. 2014;73:1232-9.

17. Wang $\mathrm{Y}$, Kahaleh $\mathrm{B}$. Epigenetic repression of bone morphogenetic protein receptor II expression in scleroderma. J Cell Mol Med. 2013;17(10):1291-9.

18. Romero L, Zhang D, Cooke J, Ho HK, Avalos E, Herrera $\mathrm{R}$, et al. Differential expression of nitric oxide by dermal microvascular endothelial cells from patients with scleroderma. Vasc Med. 2000;5:147-58.

19. Zhu H, Zhu C, Mi W, Chen T, Zhao H, Zuo X, et al. Integration of genome-wide DNA methylation and transcription uncovered aberrant methylation-regulated genes and pathways in the peripheral blood mononuclear cells of systemic sclerosis. Int J Rheumatol. 2018;2018:7342472-.

20. Ramos PS, Zimmerman KD, Haddad S, Langefeld CD, Medsger TA, Jr., Feghali-Bostwick CA. Integrative analysis of DNA methylation in discordant twins unveils distinct architectures of systemic sclerosis subsets. Clin Epigenetics. 2019;11(1):58-

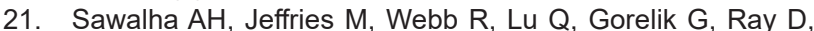
et al. Defective T-cell ERK signaling induces interferonregulated gene expression and overexpression of methylation-sensitive genes similar to lupus patients. Genes \& Immun. 2008;9(4):368-78.

22. Hellman A, Chess A. Gene body-specific methylation on the active $X$ chromosome. Science. 2007;315:1141-3.

23. Lian $X$, Xiao $R$, Hu X, Kanekura T, Jiang H, Li $Y$, et al. DNA demethylation of CD40L in CD4+ T cells from women with systemic sclerosis: A possible explanation for female susceptibility. Arthritis Rheum. 2012;64(7):2338-45.

24. Fukasawa C, Kawaguchi Y, Harigai M, Sugiura T, Takagi $\mathrm{K}$, Kawamoto M, et al. Increased CD40 expression in skin fibroblasts from patients with systemic sclerosis (SSc): role of CD40-CD154 in the phenotype of SSc fibroblasts. Eur J Immunol. 2003;33(10):2792-800.

25. Lu Q, Kaplan M, Ray D, Ray D, Zacharek S, Gutsch D, et al. Demethylation of ITGAL (CD11a) regulatory sequences in systemic lupus erythematosus. Arthritis Rheum. 2002;46(5):1282-91.

26. Jiang $\mathrm{H}$, Xiao $\mathrm{R}$, Lian X, Kanekura T, Luo $\mathrm{Y}, \mathrm{Yin} \mathrm{Y}$, et al. Demethylation of TNFSF7 contributes to CD70 overexpression in CD4+ T cells from patients with systemic sclerosis. Clin Immunol. 2012;143(1):39-44.

27. Yin $Y$, Liu $W$, Dai $Y$. SOCS3 and its role in associated diseases. Human Immunology. 2015;76(10):775-80.

28. Hill GR, Kuns RD, Raffelt NC, Don ALJ, Olver SD, Markey $\mathrm{KA}$, et al. SOCS3 regulates graft-versus-host disease. Blood. 2010;116(2):287-96.

29. Kondo Y, Shen L, Cheng AS, Ahmed S, Boumber Y, Charo $\mathrm{C}$, et al. Gene silencing in cancer by histone $\mathrm{H} 3$ lysine 27 trimethylation independent of promoter DNA methylation. Nat Genet. 2008;40(6):741-50.

30. Hemmatazad H, Rodrigues HM, Maurer B, Brentano F, Pileckyte M, Distler JHW, et al. Histone deacetylase 7, a potential target for the antifibrotic treatment of systemic sclerosis. Arthritis Rheum. 2009;60(5):1519-29.

31. Huber LC, Distler JHW, Moritz F, Hemmatazad H, Hauser T, Michel BA, et al. Trichostatin A prevents the accumulation of extracellular matrix in a mouse model of bleomycin-induced skin fibrosis. Arthritis Rheum. 2007;56(8):2755-64

32. Matouk CC, Marsden PA. Epigenetic regulation of vascular endothelial gene expression. Circ Res. 2008;102(8):873-87.

33. Ciechomska M, O'Reilly S, Przyborski S, Oakley F, BoguniaKubik K, van Laar JM. Histone demethylation and Toll-like receptor 8-dependent cross-talk in monocytes promotes transdifferentiation of fibroblasts in systemic sclerosis via Fra-2. 
Arthritis Rheum. 2016;68(6):1493-504.

34. Maurer B, Distler JHW, Distler O. The Fra-2 transgenic mouse model of systemic sclerosis. Vascul Pharmacol. 2013;58(3):194-201.

35. Krämer M, Dees C, Huang J, Schlottmann I, Palumbo-Zerr $\mathrm{K}$, Zerr $\mathrm{P}$, et al. Inhibition of $\mathrm{H} 3 \mathrm{~K} 27$ histone trimethylation activates fibroblasts and induces fibrosis. Ann Rheum Dis. 2013;72:614-20.

36. Hong S, Cho Y-W, Yu L-R, Yu H, Veenstra TD, Ge K. Identification of JmjC domain-containing UTX and JMJD3 as histone $\mathrm{H} 3$ lysine 27 demethylases. Proc Natl Acad Sci USA. 2007;104(47):18439-44.

37. Agger K, Cloos PAC, Christensen J, Pasini D, Rose S, Rappsilber J, et al. UTX and JMJD3 are histone H3K27 demethylases involved in HOX gene regulation and development. Nature. 2007;449(7163):731-4.

38. Wang $Y$, Yang $Y$, Luo $Y$, Yin $Y$, Wang Q, Li Y, et al. Aberrant histone modification in peripheral blood $B$ cells from patients with systemic sclerosis. Clin Immunol. 2013;149(1):46-54.

39. Wang Q, Xiao Y, Shi Y, Luo Y, Li Y, Zhao M, et al. Overexpression of JMJD3 may contribute to demethylation of H3K27me3 in CD4+ T cells from patients with systemic sclerosis. Clin Immunol. 2015;161(2):396-9.

40. Ambros $V$. The functions of animal microRNAs. Nature. 2004:431(7006):350-5.

41. Bartel DP. Metazoan MicroRNAs. Cell. 2018;173(1):20-51.

42. Bartel DP. MicroRNAs: Genomics, Biogenesis, Mechanism, and Function. Cell. 2004;116(2):281-97.

43. Maurer B, Stanczyk J, Jüngel A, Akhmetshina A, Trenkmann $M$, Brock M, et al. MicroRNA-29, a key regulator of collagen expression in systemic sclerosis. Arthritis Rheum. 2010;62(6):1733-43.

44. Zhu H, Li Y, Qu S, Luo H, Zhou Y, Wang Y, et al. MicroRNA expression abnormalities in limited cutaneous scleroderma and diffuse cutaneous scleroderma. J Clin Immunol. 2012;32(3):514-22.

45. Zhu H, Luo H, Li Y, Zhou Y, Jiang Y, Chai J, et al. MicroRNA-21 in scleroderma fibrosis and its function in TGF- $\beta$-regulated fibrosis-related genes expression. J Clin Immunol. 2013;33(6):1100-9.

46. Wermuth PJ, Piera-Velazquez S, Jimenez SA. Exosomes isolated from serum of systemic sclerosis patients display alterations in their content of profibrotic and antifibrotic microRNA and induce a profibrotic phenotype in cultured normal dermal fibroblasts. Clin Exp Rheumatol. 2017;35 Suppl 106(4):21-30.

47. Sing T, Jinnin M, Yamane K, Honda N, Makino K, Kajihara
I, et al. microRNA-92a expression in the sera and dermal fibroblasts increases in patients with scleroderma. Rheumatology. 2012;51(9):1550-6.

48. Honda N, Jinnin M, Kajihara I, Makino T, Makino K, Masuguchi $S$, et al. TGF- $\beta$-mediated downregulation of microRNA-196a contributes to the constitutive upregulated type I collagen expression in scleroderma dermal fibroblasts. J Immunol. 2012;188:3323-31.

49. Honda N, Jinnin M, Kira-Etoh T, Makino K, Kajihara I, Makino T, et al. miR-150 down-regulation contributes to the constitutive type I collagen overexpression in scleroderma dermal fibroblasts via the induction of integrin $\beta 3$. Am J Pathol. 2013;182(1):206-16.

50. Jiang X, Tsitsiou E, Herrick SE, Lindsay MA. MicroRNAs and the regulation of fibrosis. FEBS J. 2010;277(9):2015-21.

51. Makino K, Jinnin M, Kajihara I, Honda N, Sakai K, Masuguchi S, et al. Circulating miR-142-3p levels in patients with systemic sclerosis. Clin Exp Dermatol. 2012;37(1):34-9.

52. Christmann RB, Wooten A, Sampaio-Barros $P$, Borges $\mathrm{CL}$, Carvalho CRR, Kairalla RA, et al. miR-155 in the progression of lung fibrosis in systemic sclerosis. Arthritis Res Ther. 2016;18(1):155-.

53. Wermuth PJ, Piera-Velazquez S, Rosenbloom J, Jimenez SA. Existing and novel biomarkers for precision medicine in systemic sclerosis. Nat Rev Rheumatol. 2018;14(7):421-32.

54. Vrba L, Garbe JC, Stampfer MR, Futscher BW. Epigenetic regulation of normal human mammary cell type-specific miRNAs. Genome Res. 2011;21(12):2026-37.

55. Messemaker TC, Chadli L, Cai G, Goelela VS, Boonstra $M$, Dorjée $A L$, et al. Antisense long non-coding RNAs are deregulated in skin tissue of patients with systemic sclerosis. J Invest Dermatol. 2018;138(4):826-35.

56. Wang Z, Jinnin M, Nakamura K, Harada M, Kudo $H$, Nakayama W, et al. Long non-coding RNA TSIX is upregulated in scleroderma dermal fibroblasts and controls collagen mRNA stabilization. Exp Dermatol. 2016;25(2):131-6.

57. Xu F, Jin L, Jin $Y$, Nie Z, Zheng $H$. Long noncoding RNAs in autoimmune diseases. J Biomed Mater Res A. 2019;107(2):468-75

58. Wu G-C, Pan H-F, Leng R-X, Wang D-G, Li X-P, Li X-M, et al. Emerging role of long noncoding RNAs in autoimmune diseases. Autoimmun Rev. 2015;14(9):798-805.

59. Colletti M, Galardi A, De Santis M, Guidelli GM, Di Giannatale A, Di Luigi L, et al. Exosomes in systemic sclerosis: messengers between immune, vascular and fibrotic components? Int J Mol Sci. 2019;20(18):4337. 